\title{
The Association between Limited English Proficiency and Sepsis Mortality
}

\author{
Zachary G Jacobs*, MD, Priya A Prasad, PhD, MPH, Margaret C Fang, MD, MPH, \\ Yumiko Abe-Jones, MS, Kirsten N Kangelaris, MD, MAS*
}

Division of Hospital Medicine, University of California, San Francisco, San Francisco, California.

BACKGROUND: Limited English proficiency (LEP) has been implicated in poor health outcomes. Sepsis is a frequently fatal syndrome that is commonly encountered in hospital medicine. The impact of LEP on sepsis mortality is not currently known.

OBJECTIVE: To determine the association between LEP and sepsis mortality.

DESIGN: Retrospective cohort study.

SETTING: 800-bed, tertiary care, academic medical center.

PATIENTS: Electronic health record data were obtained for adults admitted to the hospital with sepsis between June 1, 2012 and December 31, 2016.

MEASUREMENTS: The primary predictor was LEP. Patients were defined as having LEP if their self-reported primary language was anything other than English and interpreter services were required during hospitalization. The primary outcome was inpatient mortality. Mortality was compared across races stratified by LEP using chisquared tests of significance. Bivariable and multivariable logistic regressions were performed to investigate the association between mortality, race, and LEP, adjusting for baseline characteristics, comorbidities, and illness severity.

RESULTS: Among 8,974 patients with sepsis, we found that 1 in 5 had LEP, $62 \%$ of whom were Asian. LEP was highly associated with death across all races except those identifying as Black and Latino. LEP was associated with a $31 \%$ increased odds of mortality after adjusting for illness severity, comorbidities, and other baseline characteristics, including race (OR 1.31, 95\% $\mathrm{Cl} 1.06-1.63, P=.02)$.

CONCLUSIONS: In a single-center study of patients hospitalized with sepsis, LEP was associated with mortality across nearly all races. This is a novel finding that will require further exploration into the causal nature of this association. Journal of Hospital Medicine 2020;15:140146. (C) 2020 Society of Hospital Medicine epsis is defined as a life-threatening organ dysfunction that occurs in response to systemic infection. ${ }^{1,2}$ It is frequently fatal, common in hospital medicine, and a leading contributor to critical illness, morbidity, and healthcare expenditures. ${ }^{2-5}$ While sepsis care and outcomes have improved in the past decade, ${ }^{6,7}$ inpatient mortality remains high. ${ }^{8}$

A number of studies have sought to determine whether race plays a role in sepsis mortality. While Black patients with sepsis have frequently been identified as having the highest rates of death, ${ }^{9-14}$ similar observations have been made for most nonWhite races/ethnicities. ${ }^{13-15}$ Studies have also demonstrated higher rates of hospital-acquired infections among Asian and Latino patients. ${ }^{16}$

There are several possible explanations for why racial minorities experience disparate outcomes in sepsis, including

*Corresponding Authors: Zachary G. Jacobs, MD; E-mail: JacobsZ@ohsu.edu; Telephone: 503-418-0420; Twitter: @ZacharyGJacobs. Kirsten N. Kangelaris, MD, MS; E-mail: Kirsten.Kangelaris@ucsf.edu; Telephone: 415-476-4852; Twitter: @KKangelaris

Published online first November 20, 2019

Find Additional Supporting Information in the online version of this article.

Received: May 18, 2019; Revised: September 16, 2019;

Accepted: September 23, 2019

() 2020 Society of Hospital Medicine DOI 10.12788/jhm.3334 access to care, comorbidities, implicit biases, and biological or environmental factors, ${ }^{17-20}$ as well as characteristics of hospitals most likely to care for racial minorities. . $13,15,21^{\text {One explanation }}$ that has not been explored is that racial disparities in sepsis are mediated by language. Limited English proficiency (LEP) has previously been associated with increased rates of adverse hospital events, ${ }^{22}$ longer length of stay, ${ }^{23}$ and greater likelihood of readmission. ${ }^{24}$ LEP has also been shown to represent a significant barrier to accessing healthcare and preventive screening. ${ }^{25}$ The role of LEP in sepsis mortality, however, has yet to be examined.

The diverse patient population at the University of California, San Francisco (UCSF) provides a unique opportunity to build upon existing literature by further exploring racial differences in sepsis, specifically by investigating the role of LEP. The objective of this study was to determine the association between LEP and inpatient mortality among adults hospitalized with sepsis.

\section{METHODS}

\section{Setting}

The study was conducted at the University of California, San Francisco, California (UCSF), an 800-bed tertiary care, academic medical center. It was approved by the UCSF Institutional Review Board with waiver of informed consent. UCSF cares for a population of patients who are racially and linguistical- 


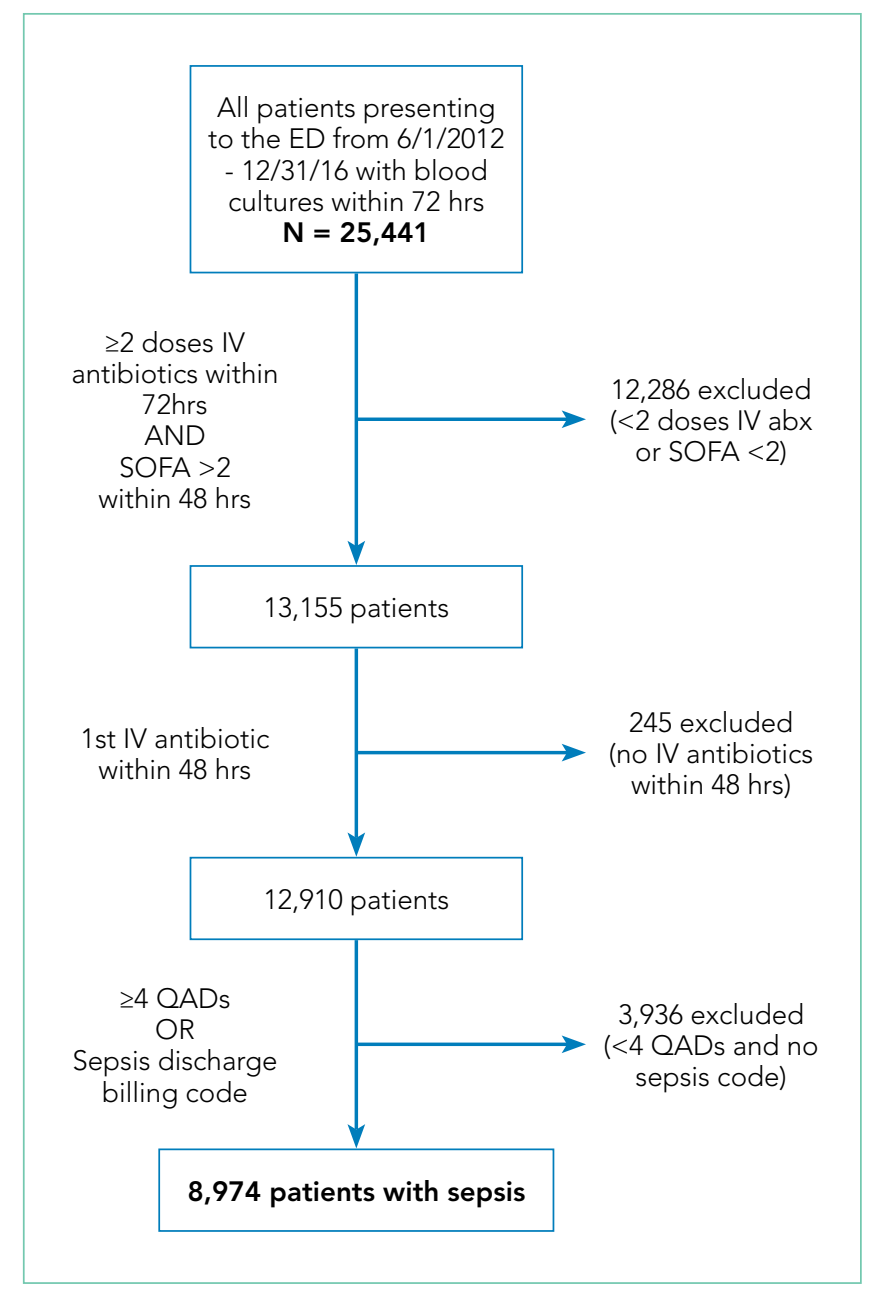

FIG. Identification of study cohort of patients hospitalized with sepsis.

Abbreviation: ED, emergency department; IV, intravenous; LEP, limited English proficiency; $\mathrm{QAD}$, qualifying antibiotic day; SOFA, sequential (sepsis-related) organ failure assessment.

ly diverse, with high proportions of patients of East Asian descent and with LEP. According to recent United States census estimates, more than half of San Francisco County residents identify as non-White (35\% Asians, 15\% Hispanic/Latino, 6\% Black), and $44 \%$ report speaking a language other than English at home. ${ }^{26}$

\section{Study Population and Data Collection}

The UCSF Medical Center uses the electronic health record (EHR) Epic (Epic 2017, Epic Systems Corporation, Verona, Wisconsin). We obtained computerized EHR data from Clarity, the relational database that stores Epic's inpatient data in thousands of tables. We identified all patients $\geq 18$ years of age presenting to the emergency department (ED) between June 1, 2012 and December 31, 2016 with suspected serious infection, defined as having blood cultures ordered within 72 hours of ED presentation $(\mathrm{N}=25,441)$. Patients who did not receive at least two doses of intravenous (IV) antibiotics within 48 hours were excluded, as they were unlikely to have serious infections.

We defined sepsis based on Sepsis-3 consensus guidelines $^{2}$ as a change in sequential [sepsis-related] organ failure assessment (SOFA) score $\geq 2$ within the first 48 hours of ED presentation. The SOFA score is comprised of six variables representing different organ systems, each rated 0-4 based on the degree of dysfunction. ${ }^{2}$ Patient vital signs, laboratory data, vasopressor medication doses, and ventilator settings were used to determine the exact timestamp at which each patient attained a change in SOFA score $\geq 2$. Missing values were considered to be normal. To adjust for baseline organ dysfunction, SOFA elements associated with elevated bilirubin and/or creatinine were excluded for patients with chronic liver/kidney disease based on Elixhauser comorbidities. ${ }^{27} \mathrm{We}$ chose to focus on the first 48 hours in an attempt to capture patients with the most severe illnesses and the highest probability of true sepsis.

All primary and secondary International Classification of Diseases (ICD)-9/10 diagnosis codes were extracted from Clarity coding tables at the time of hospital discharge. Diagnosis codes signifying bacterial infection were grouped into the following categories based on type/location: pneumonia; bacteremia; urinary tract infection; and skin and soft tissue infection. All remaining diagnostic codes indicating bacterial infections at other sites were categorized as "Other". If no codes indicating infection were present, patients were categorized as "None coded". Patients with discharge diagnosis codes of "sepsis" were also identified. Dates and times of antibiotic administrations were obtained from the medications table. Time to first antibiotic was defined as the time in minutes from ED presentation to initiation of the first IV antibacterial medication. This variable was transformed using a natural log transformation based on best fit for normal distribution.

We limited our analyses to 8,974 patients who were diagnosed with sepsis as defined above and had either (1) $\geq 4$ qualifying antibiotic days (QADs) or (2) an ICD-9/10 discharge diagnosis code of "sepsis" (Figure). QADs were defined based on the recent publication by Rhee et al. as having received four or more consecutive days of antibiotics, with the first dose given IV within 48 hours of presentation. ${ }^{28}$ Patients who died or were discharged to hospice prior to the 4th QAD were also included. These additional parameters were added to increase specificity of the study sample for patients with true sepsis. Patients admitted to all levels of care (acute care, transitional care unit [TCU], intensive care unit [ICU]) and under all hospital services were included. There were no missing data for mortality, race, or language. We chose to focus on patients with sepsis in this initial study as this is a common diagnosis in hospital medicine that is enriched for high mortality.

\section{Primary Outcome}

The primary outcome of the study was inpatient mortality, which was obtained from the hospital encounters table in Clarity.

\section{Primary Predictors}

The primary predictor of interest was LEP. The encounter numbers from the dataset were used to link to self-reported demographic data, including "preferred language" and need for interpreter services. A manual chart review of 60 patients 
speaking the top six languages was conducted to verify the accuracy of the data on language and interpreter use (KNK). Defining the gold standard for LEP as having any chart note indicating non-English language and/or that an interpreter was used, the "interpreter needed" variable in Epic was found to have a positive predictive value for LEP of $100 \%$. Therefore, patients in the study cohort were defined as having LEP if they met both of the following criteria: (1) a self-reported "preferred language" other than English and (2) having the "interpreter needed" variable indicating "yes".

\section{Covariate Data Collection}

Additional data were obtained from the demographics tables, including age, race, sex, and insurance status. Race and ethnicity were combined into a single five-category variable including White, Asian, Black, Latino, and Other. This approach has been suggested as the best way to operationalize these variables $^{29}$ and has been utilized by similar studies in the literature. $9,14,15$ We considered the Asian race to include all people of East Asian, Southeast Asian, or South Asian descent, which is consistent with the United States Census Bureau definition. ${ }^{30}$ Patients identifying as Native Hawaiians/Pacific Islanders, $\mathrm{Na}$ tive Americans/Alaskan Natives, as well as those with unspecified race or ethnicity, were categorized as Other. Insurance status was categorized as Commercial, Medicare, Medicaid, or Other.

We estimated illness severity in several ways. First, the total qualifying SOFA score was calculated for each patient, which was defined as the total score achieved at the time that SOFA criteria were first met ( $\geq 2$, within 48 hours). Second, we dichotomized patients based on whether they had received mechanical ventilation at any point during hospitalization. Finally, we used admission location as a surrogate marker for severity at the time of initial hospitalization.

To estimate the burden of baseline comorbidities, we calculated the van Walraven score (WWS), ${ }^{31}$ a validated modification of the Elixhauser Comorbidity Index. ${ }^{27}$ This score conveys an estimated risk of in hospital death based on ICD-9/10 diagnosis codes for preexisting conditions, which ranges from $<1 \%$ for the minimum score of -19 to $>99 \%$ for the maximum score of 89 .

\section{Statistical Analyses}

All statistical analyses were performed using Stata software version 15 (StataCorp LLC, College Station, Texas). Baseline demographics and patient characteristics were stratified by LEP. These were compared using two-sample t-tests or chisquared tests of significance. Wilcoxon rank-sum tests were used for non-normally distributed variables. Inpatient mortality was compared across all races stratified by LEP using chisquared tests of significance.

We fit a series of multivariable logistic regression models to examine the association between race and inpatient mortality adjusting for LEP and other patient/clinical characteristics. We first examined the unadjusted association between mortality and race; then adjusted for LEP alone; and finally adjusted for all covariates of interest, including LEP, age, sex, insurance status, year, admission level of care, VWS, total qualifying SOFA score, need for mechanical ventilation, site of infection, and time to first IV antibiotic. A subgroup analysis was also performed using the fully adjusted model restricted to patients who were mechanically ventilated. This population was selected because the patients (1) have among the highest severity of illness and (2) share a common barrier to communication, regardless of English proficiency.

Several potential interactions between LEP with other covariates were explored, including age, race, ICU admission level of care, and need for mechanical ventilation. Lastly, a mediation analysis was performed based on Baron \& Kenny's four-step mode ${ }^{32}$ in order to calculate the proportion of the association between race and mortality explained by the proposed mediator (LEP).

To evaluate for the likelihood of residual confounding, we calculated an E-value, which is defined as the minimum strength of association that an unmeasured confounder would need to have with both the predictor and outcome variables, above and beyond the measured covariates, in order to fully explain away an observed predictor-outcome association. ${ }^{33,34}$

\section{RESULTS}

We identified 8,974 patients hospitalized with sepsis based on the above inclusion criteria. This represented a medically complex, racially and linguistically diverse population (Table 1). The cohort was comprised of $24 \%$ Asian, $12 \%$ Black, and $11 \%$ Latino patients. Among those categorized as Other race, $\mathrm{Na}$ tive Americans/Alaskan Natives and Native Hawaiians/Pacific Islanders accounted for $4 \%(n=31)$ and $21 \%(n=159)$, respectively. A fifth of all patients had LEP ( $n=1,716), 62 \%$ of whom were Asian ( $n=1,064)$. Patients with LEP tended to be older, female, and to have a greater number of comorbid conditions (Table 1). The total qualifying SOFA score was also higher among patients with LEP (median 5; interquartile range [IQR]: 4-8 vs 5; IQR: $3-7 ; P<.001)$, though there was no association between LEP and mechanical ventilation $(P=.22)$. The prevalence of LEP differed significantly across races, with 50\% LEP among Asians, 32\% among Latinos, 5\% among White patients $(P<$ .001). Only eight Black patients had LEP. More than 40 unique languages were represented in the cohort, with English, Cantonese, Spanish, Russian, and Mandarin accounting for $~ 95 \%$ (Appendix Table 1). Among Latino patients, 63\% spoke English and $36 \%$ spoke Spanish.

In-hospital mortality was significantly higher among patients who had LEP ( $n=268 / 1,716,16 \%$ ) compared to non-LEP patients ( $n=678 / 7,258,9 \%$ ), with $80 \%$ greater unadjusted odds of mortality (OR 1.80; 95\% Cl: 1.54-2.09; P<.001). Notably we also found that Asian race was associated with a 1.57 unadjusted odds of mortality compared to White race (95\% Cl: 1.34-1.85; $P<$.001). Age, WWS, total qualifying SOFA score, mechanical ventilation, and admission level of care all exhibited a positive dose-response association with mortality (Appendix Table 2). In unadjusted analyses, there was no evidence of interaction between LEP and age $(P=.38)$, LEP and race $(P=.45)$, LEP 
TABLE 1. Baseline Patient and Clinical Characteristics of 8,974 Patients Hospitalized with Sepsis Stratified by LEP

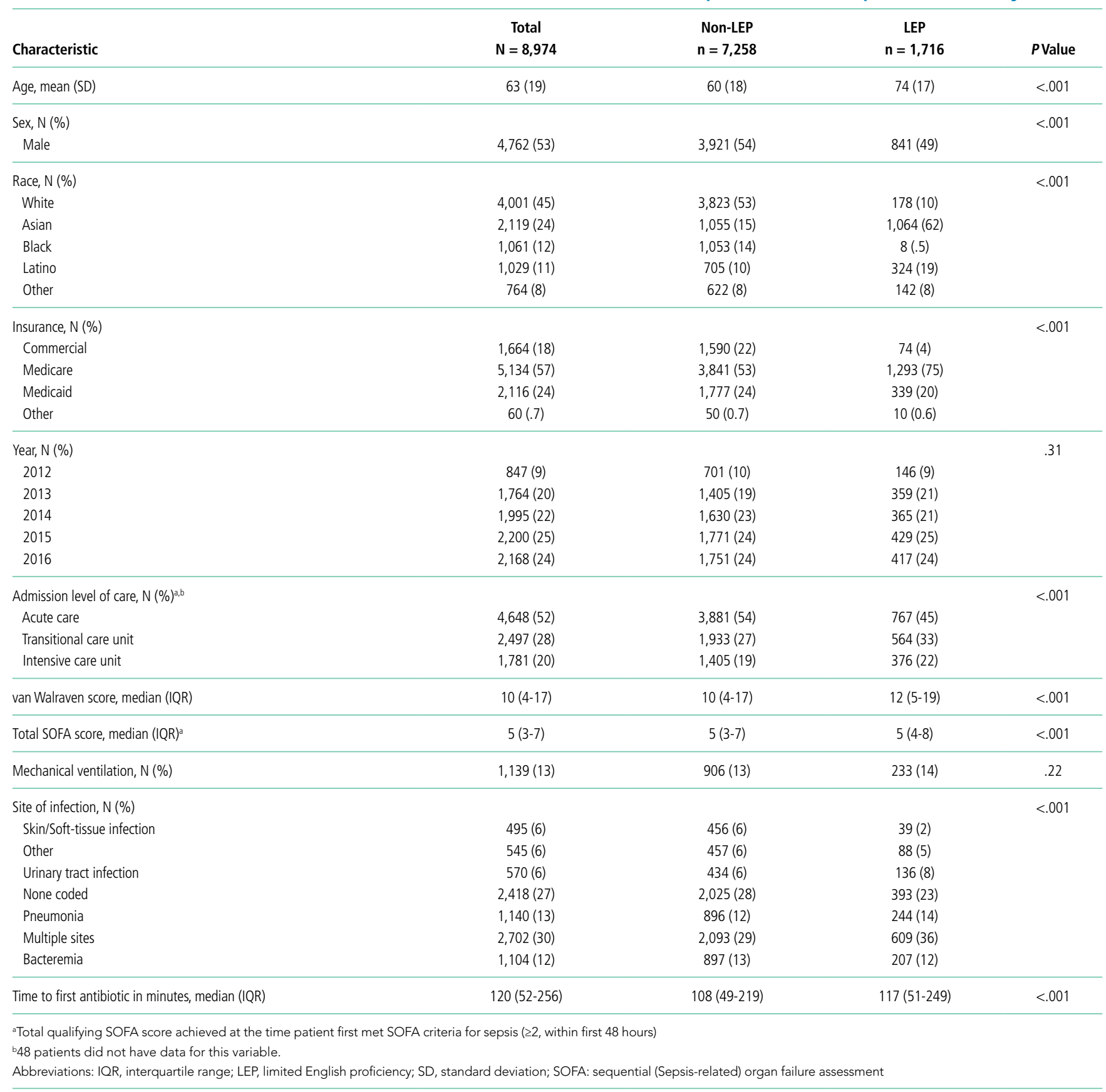

and ICU admission level of care $(P=.31)$, or LEP and mechanical ventilation $(P=.19)$. Asian patients had the highest overall mortality (14\% total, $17 \%$ with LEP). LEP was associated with increased unadjusted mortality among White, Asian, and Other races compared to their non-LEP counterparts (Appendix Figure 1). There was no significant difference in mortality between Latino patients with and without LEP. The sample size for Black patients with LEP $(n=8)$ was too small to draw conclusions about mortality.

Following multivariable logistic regression modeling for the association between race and mortality, we found that the increased odds of death among Asian patients was partially attenuated after adjusting for LEP (odds ratio [OR] 1.23, 95\% Cl: 1.02-1.48; $P=.03$; Table 2). Meanwhile, LEP was associated with a 1.66 odds of mortality (95\% Cl: 1.38-1.99; $P<.001)$ after adjustment for race. In the full multivariable model adjusting for demographics and clinical characteristics, illness severity, and comorbidities, LEP was associated with a $31 \%$ increase in the odds of mortality compared to non-LEP (95\% Cl: 1.06-1.63; $P=.02)$. In this model, the association between Asian race and mortality was now fully attenuated, with a point estimate near 1.0 (OR 0.98; 95\% Cl: 0.79-1.22; $P=.87$ ). Markers of illness se- 
TABLE 2. Sequential Multivariable Modeling of the Association between Race/Ethnicity and Inpatient Mortality Adjusted for LEP and Other Covariates ${ }^{a}$

\begin{tabular}{|c|c|c|c|c|c|c|}
\hline \multirow[b]{3}{*}{ Covariate } & \multicolumn{2}{|c|}{$\begin{array}{l}\text { Model 1: } \\
\text { Unadjusted }\end{array}$} & \multicolumn{2}{|c|}{$\begin{array}{l}\text { Model 2: } \\
\text { Adjusting for LEP }\end{array}$} & \multicolumn{2}{|c|}{$\begin{array}{c}\text { Model 3: } \\
\text { Adjusting for LEP + Other Covariates }\end{array}$} \\
\hline & Mortality & & Mortality & & Mortality & \\
\hline & OR $(95 \% \mathrm{Cl})$ & $P$ Value & OR $(95 \% \mathrm{Cl})$ & $P$ Value & OR $(95 \% \mathrm{Cl})$ & $P$ Value \\
\hline \multicolumn{7}{|c|}{ Race/Ethnicity } \\
\hline White & ref. & ref. & ref. & ref. & ref. & ref. \\
\hline Asian & $1.57(1.34-1.85)$ & $<.001$ & $1.23(1.02-1.48)$ & .03 & $0.98(0.79-1.22)$ & .87 \\
\hline Black & $0.90(0.71-1.15)$ & .40 & $0.92(0.73-1.17)$ & .51 & $0.72(0.55-0.96)$ & .03 \\
\hline Latino & $0.82(0.64-1.06)$ & .12 & $0.70(0.54-0.91)$ & .01 & $0.84(0.63-1.13)$ & .25 \\
\hline Other & $1.43(1.13-1.81)$ & .003 & $1.32(1.04-1.68)$ & .02 & $1.05(0.80-1.39)$ & .72 \\
\hline \multicolumn{7}{|l|}{ Language } \\
\hline Non-LEP & - & - & ref. & ref. & ref. & ref. \\
\hline LEP & - & - & $1.66(1.38-1.99)$ & $<.001$ & $1.31(1.06-1.63)$ & .02 \\
\hline \multicolumn{7}{|c|}{ ancluding age, sex, insurance, year, admission level of care, van Walraven score, total SOFA score ${ }^{b}$, need for mechanical ventilation, site of infection, and time to first intravenous antibiot } \\
\hline \multicolumn{7}{|c|}{ 'Total qualifying SOFA score achieved at the time patient first met sepsis definition SOFA criteria ( $\geq 2$, within first 48 hours) } \\
\hline \multicolumn{7}{|c|}{ `Natural log transformed for best fit normal distribution } \\
\hline \multicolumn{7}{|c|}{ Abbreviations: LEP, limited English proficiency; OR, odds ratio; SOFA, sequential (sepsis-related) organ failure assessment } \\
\hline
\end{tabular}

verity, including total qualifying SOFA score (OR 1.23; $95 \% \mathrm{Cl}$ : $1.20-1.27 ; P<.001)$ and need for mechanical ventilation (OR 1.88; $95 \% \mathrm{Cl}: 1.52-2.33 ; P<.001)$, were both associated with greater odds of death. Based on a four-step mediation analysis, LEP was found to be a partial mediator to the association between Asian race and mortality (76\% proportion explained). The E-value for the association between LEP and mortality was 1.95 , with an E-value for the corresponding confidence interval of 1.29 .

In a subgroup analysis using the fully adjusted model restricted to patients who were mechanically ventilated during hospitalization, the association between LEP and mortality was no longer present (OR 1.15; 95\% Cl: 0.76-1.72; $P=.51$ ).

\section{DISCUSSION}

At a single US academic medical center serving a diverse population, we found that LEP was associated with sepsis mortality across all races except Black and Latino, conveying a $31 \%$ increase in the odds of death after adjusting for illness severity, comorbidities, and baseline characteristics. The higher mortality among Asian patients was largely mediated by LEP (76\% proportion explained). While previous studies have variably found Black, Asian, Latino, and other non-White races/ethnicities to be at an increased risk of death from sepsis, ${ }^{9-15}$ LEP has not been previously evaluated as a mediator of sepsis mortality. We were uniquely suited to uncover such an association due to the racial and linguistic diversity of our patient population. LEP has previously been implicated in poor health outcomes among hospitalized patients in general.22-24 Future studies will be necessary to determine whether similar associations between LEP and mortality are observed among broader patient populations outside of sepsis.

There are a number of possible explanations for how LEP could mediate the association between race and mortality. First, LEP is known to be associated with greater difficulties in accessing medical care, ${ }^{25}$ which could result in poorer baseline control of chronic comorbid conditions, fewer opportunities for preventive screening, and greater reluctance to seek medical attention when ill, theoretically leading to more severe presentations and worse outcomes. Indeed, LEP patients in our cohort had both a shorter median time to receiving their first antibiotic, as well as a higher total qualifying SOFA score, both of which may suggest more severe initial presentations. LEP is also known to contribute to, or exacerbate, the impact of low health literacy, which is itself associated with poor health. ${ }^{35}$ Second, implicit biases may also have been present, as they are known to be common among healthcare providers and have been shown to negatively impact patient care. ${ }^{36}$

Finally, it is possible that the association is related to the language barrier itself, which impacts providers' ability to take an appropriate clinical history, and can lead to clinical errors or delays in care. ${ }^{37}$ The fact that the association between LEP and mortality was eliminated when the analysis was restricted to mechanically ventilated patients seems to support this, since differences in language proficiency become irrelevant in this subgroup. While we are unable to comment on causality based on this observational study, we included a directed acyclic graph (DAG) in the supplemental materials, which shows one proposed model for describing these associations (Appendix Figure 2).

Assuming that the language barrier itself does, at least in part, drive the observed association, LEP represents a potentially modifiable risk factor that could be a target for quality improvement interventions. There is evidence that the use of medical interpreters among patients with LEP leads to greater satisfaction, fewer errors, and improved clinical outcomes; ${ }^{38}$ 
however, several recent studies have documented underutilization of professional interpreter services, even when readily available. ${ }^{39,40}$ At our institution, phone and video interpreter services are available 24/7 for approximately 150 languages. Due to limitations inherent to the EHR, we were unable to ascertain the extent to which these services were used in the present study. Heavy clinical workloads, connectivity issues, and missing or faulty equipment represent theoretical barriers to utilization of these services.

There are some limitations to our study. First, by utilizing a large database of electronic data, the quality of our analyses was reliant on the accuracy of the EHR. Demographic data such as language may have been subject to misclassification due to self-reporting. We attempted to minimize this by also including the need for interpreter services within the definition of LEP, which was validated by manual chart review. Second, generalizability is limited in this single-center study conducted at an institution with unique demographics, wherein nearly twothirds of the LEP patients were Asian, and the Chinese-speaking population outnumbered those who speak Spanish.

Finally, the most important limitation to our study is the potential for residual confounding. While we attempted to mitigate this by adjusting for as many clinically relevant covariates as possible, there may still be unmeasured confounders to the association between LEP and mortality, such as access to outpatient care, functional status, interpreter use, and other markers of illness severity like the number and type of supportive therapies received. Based on our E-value calculations, with an observed OR of 1.31 for the association between LEP and mortality, an unmeasured confounder with an OR of 1.95 would fully explain away this association, while an OR of 1.29 would shift the confidence interval to include the null. These values suggest at least some risk of residual confounding. The fact that our fully adjusted model included multiple covariates, including several markers of illness severity, does somewhat lessen the likelihood of a confounder achieving these values, since they represent the minimum strength of an unmeasured confounder above and beyond the measured covariates. Regardless, the finding that patients with LEP are more likely to die from sepsis remains an important one, recognizing the need for further studies including multicenter investigations.

In this study, we showed that LEP was associated with sepsis mortality across nearly all races in our cohort. While Asian race was associated with a higher unadjusted odds of death compared to White race, this was attenuated after adjusting for LEP. This may suggest that some of the racial disparities in sepsis identified in prior studies were in fact mediated by language proficiency. Further studies will be required to explore the causal nature of this novel association. If modifiable factors are identified, this could represent a potential target for future quality improvement initiatives aimed at improving sepsis outcomes.

Disclosures: The authors have no disclosures to declare

Funding: This study was supported in part by the National Heart, Lung, and Blood Institute of the National Institutes of Health (Grant 1K24HL141354 to Dr.
Fang, and grant 1K23HL116800 to Dr. Kangelaris). Dr. Prasad was supported by the National Center for Advancing Translational Sciences, National Institutes of Health, through UCSF-CTSI Grant Number \#A127552. Data acquisition for this publication was supported by UCSF Academic Research Systems, and by the National Center for Advancing Translational Sciences, National Institutes of Health, through UCSF-CTSI Grant Number UL1 TR001872.

Disclaimer: The contents are solely the responsibility of the authors and do not necessarily represent the official views of the University of California, San Francisco or the National Institutes of Health.

\section{References}

1. De Backer DD, Dorman T. Surviving sepsis guidelines: A continuous move toward better care of patients with sepsis. JAMA. 2017;317(8):807-808. https://doi.org/10.1001/jama.2017.0059.

2. Singer $M$, Deutschman CS, Seymour CW, et al. The third international consensus definitions for sepsis and septic shock (Sepsis-3). JAMA. 2016;315(8):801-810. https://doi.org/10.1001/jama.2016.0287.

3. Angus DC, Linde-Zwirble WT, Lidicker J, et al. Epidemiology of severe sepsis in the United States: analysis of incidence, outcome, and associated costs of care. Crit Care Med. 2001;29(7):1303-1310. https://doi.org/10.1097/00003246200107000-00002.

4. Mayr FB, Yende S, Angus DC. Epidemiology of severe sepsis. Virulence. 2014;5(1):4-11. https://doi.org/10.4161/viru.27372.

5. Dellinger RP, Levy MM, Rhodes A, et al. Surviving Sepsis Campaign: international guidelines for management of severe sepsis and septic shock: 2012. Crit Care Med. 2013;41(2):580-637. https://doi.org/10.1097/ CCM.0b013e31827e83af.

6. Levy MM, Rhodes A, Phillips GS, et al. Surviving Sepsis Campaign: association between performance metrics and outcomes in a 7.5-year study. Crit Care Med. 2015;43(1):3-12. https://doi.org/10.1097/CCM.0000000000000723.

7. Damiani E, Donati A, Serafini G, et al. Effect of performance improvement programs on compliance with sepsis bundles and mortality: a systematic review and meta-analysis of observational studies. PLOS ONE. 2015;10(5):e0125827. https://doi.org/10.1371/journal.pone.0125827.

8. Paoli CJ, Reynolds MA, Sinha M, Gitlin M, Crouser E. Epidemiology and costs of sepsis in the United States-an analysis based on timing of diagnosis and severity level. Crit Care Med. 2018;46(12):1889-1897. https://doi.org/10.1097/ CCM.0000000000003342.

9. Barnato $A E$, Alexander SL, Linde-Zwirble WT, Angus DC. Racial variation in the incidence, care, and outcomes of severe sepsis: analysis of population, patient, and hospital characteristics. Am J Respir Crit Care Med [patient]. 2008;177(3):279-284. https://doi.org/10.1164/rccm.200703-4800C.

10. Mayr FB, Yende $S$, Linde-Zwirble WT, et al. Infection rate and acute organ dysfunction risk as explanations for racial differences in severe sepsis. JAMA. 2010;303(24):2495-2503. https://doi.org/10.1001/jama.2010.851.

11. Dombrovskiy VY, Martin AA, Sunderram J, Paz HL. Occurrence and outcomes of sepsis: influence of race. Crit Care Med. 2007;35(3):763-768. https://doi. org/10.1097/01.CCM.0000256726.80998.BF.

12. Yamane D, Huancahuari N, Hou P, Schuur J. Disparities in acute sepsis care: a systematic review. Crit Care. 2015;19(Suppl 1):22. https://doi.org/10.1186/ cc14102.

13. Martin GS, Mannino DM, Eaton S, Moss M. The epidemiology of sepsis in the United States from 1979 through 2000. N Engl J Med. 2003;348(16):15461554. https://doi.org/10.1056/NEJMoa022139

14. Melamed A, Sorvillo FJ. The burden of sepsis-associated mortality in the United States from 1999 to 2005: an analysis of multiple-cause-of-death data. Crit Care. 2009;13(1):R28. https://doi.org/10.1186/cc7733.

15. Jones JM, Fingar KR, Miller MA et al. Racial disparities in sepsis-related in-hospital mortality: using a broad case capture method and multivariate controls for clinical and hospital variables, 2004-2013. Crit Care Med. 2017;45(12):e1209-e1217. https://doi.org/10.1097/CCM.0000000000002699.

16. Bakullari A, Metersky ML, Wang $Y$, et al. Racial and ethnic disparities in healthcare-associated infections in the United States, 2009-2011. Infect Control Hosp Epidemiol. 2014;35(S3):S10-S16. https://doi.org/10.1086/677827.

17. Institute of Medicine. Unequal Treatment: What Healthcare Providers Need to Know about Racial and Ethnic Disparities in Healthcare. Washington, DC: National Academy Press; 2002.

18. Vogel TR. Update and review of racial disparities in sepsis. Surg Infect. 2012;13(4):203-208. https://doi.org/10.1089/sur.2012.124.

19. Esper AM, Moss M, Lewis CA, et al. The role of infection and comorbidity: factors that influence disparities in sepsis. Crit Care Med. 2006;34(10):25762582. https://doi.org/10.1097/01.CCM.0000239114.50519.0E. 
20. Soto GJ, Martin GS, Gong MN. Healthcare disparities in critical illness. Crit Care Med. 2013;41(12):2784-2793. https://doi.org/10.1097/CCM.0b013e3182a84a43.

21. Taylor SP, Karvetski CH, Templin MA, Taylor BT. Hospital differences drive antibiotic delays for black patients compared with white patients with suspected septic shock. Crit Care Med. 2018;46(2):e126-e131. https://doi. org/10.1097/CCM.0000000000002829.

22. Divi C, Koss RG, Schmaltz SP, Loeb JM. Language proficiency and adverse events in US hospitals: a pilot study. Int J Qual Health Care. 2007;19(2):60-67. https://doi.org/10.1093/intqhc/mzl069.

23. John-Baptiste A, Naglie G, Tomlinson G, et al. The effect of English language proficiency on length of stay and in-hospital mortality. J Gen Intern Med. 2004;19(3):221-228. https://doi.org/10.1111/j.1525-1497.2004.21205.x.

24. Karliner LS, Kim SE, Meltzer DO, Auerbach AD. Influence of language barriers on outcomes of hospital care for general medicine inpatients. J Hosp Med. 2010;5(5):276-282. https://doi.org/10.1002/jhm.658.

25. Hacker K, Anies M, Folb BL, Zallman L. Barriers to health care for undocumented immigrants: a literature review. Risk Manag Healthc Policy. 2015;8:175-183. https://doi.org/10.2147/RMHP.S70173.

26. QuickFacts: San Francisco County, California. U.S. Census Bureau (2016) https://www.census.gov/quickfacts/fact/table/sanfranciscocountycalifornia/ RHI425216. Accessed May 15, 2018.

27. Moore BJ, White S, Washington R, Coenen N, Elixhauser A. Identifying increased risk of readmission and in-hospital mortality using hospital administrative data: The AHRQ Elixhauser comorbidity index. Med Care. 2017;55(7):698-705. https://doi.org/10.1097/MLR.0000000000000735.

28. Rhee C, Dantes R, Epstein L, et al. Incidence and trends of sepsis in us hospitals using clinical vs claims data, 2009-2014. JAMA. 2017;318(13):1241-1249. https://doi.org/10.1001/jama.2017.13836.

29. Howell J, Emerson MO, So M. What "should" we use? Evaluating the impact of five racial measures on markers of social inequality. Sociol Race Ethn. 2017:3(1):14-30. https://doi.org/10.1177/2332649216648465.

30. Reeves T, Claudett B. United States Census Bureau. Asian Pac Islander Popul. March 2002;2003

31. van Walraven C, Austin PC, Jennings A, Quan H, Forster AJ. A modifica- tion of the Elixhauser comorbidity measures into a point system for hospital death using administrative data. Med Care. 2009;47(6):626-633. https://doi. org/10.1097/MLR.0b013e31819432e5.

32. Baron RM, Kenny DA. The moderator-mediator variable distinction in social psychological research: conceptual, strategic, and statistical considerations. J Pers Soc Psychol. 1986;51(6):1173-1182. https://doi.org/10.1037//00223514.51.6.1173.

33. VanderWeele TJ, Ding P. Sensitivity analysis in observational research: introducing the E-value. Ann Intern Med. 2017;167(4):268-274. https://doi. org/10.7326/M16-2607.

34. Mathur MB, Ding P, Riddell CA, VanderWeele TJ. Website and R package for computing E-values. Epidemiology. 2018;29(5):e45-e47. https://doi. org/10.1097/EDE.0000000000000864.

35. Sentell T, Braun KL. Low Health Literacy, Limited English proficiency, and health status in Asians, Latinos, and other racial/ethnic groups in California. J Health Commun. 2012;17 Supplement 3:82-99. https://doi.org/10.1080/1081 0730.2012 .712621

36. FitzGerald C, Hurst S. Implicit bias in healthcare professionals: a systematic review. BMC Med Eth. 2017;18(1):19. https://doi.org/10.1186/s12910-0170179-8.

37. Flores $\mathrm{G}$. The impact of medical interpreter services on the quality of health care: A systematic review. Med Care Res Rev. 2005;62(3):255-299. https://doi. org/10.1177/1077558705275416.

38. Karliner LS, Jacobs EA, Chen AH, Mutha S. Do professional interpreters improve clinical care for patients with limited English proficiency? A systematic review of the literature. Health Serv Res. 2007;42(2):727-754. https://doi. org/10.1111/j.1475-6773.2006.00629.x.

39. Diamond LC, Schenker Y, Curry L, Bradley EH, Fernandez A. Getting by: underuse of interpreters by resident physicians. J Gen Intern Med. 2009;24(2):256-262. https://doi.org/10.1007/s11606-008-0875-7.

40. López L, Rodriguez F, Huerta D, Soukup J, Hicks L. Use of interpreters by physicians for hospitalized limited English proficient patients and its impact on patient outcomes. J Gen Intern Med. 2015;30(6):783-789. https://doi. org/10.1007/s11606-015-3213-x. 